\title{
Radial transport of radiation belt electrons due to stormtime Pc5 waves
}

\author{
A. Y. Ukhorskiy, M. I. Sitnov, K. Takahashi, and B. J. Anderson \\ The Johns Hopkins University, Applied Physics Laboratory, Johns Hopkins Road, Laurel, MD, USA
}

Received: 20 August 2008 - Revised: 31 March 2009 - Accepted: 14 April 2009 - Published: 13 May 2009

\begin{abstract}
During geomagnetic storms relativistic electron fluxes in the outer radiation belt exhibit dynamic variability over multiple orders of magnitude. This requires radial transport of electrons across their drift shells and implies violation of their third adiabatic invariant. Radial transport is induced by the interaction of the electron drift motion with electric and magnetic field fluctuations in the ULF frequency range. It was previously shown that solar-wind driven ULF waves have long azimuthal wave lengths and thus can violate the third invariant of trapped electrons in the process of resonant interaction with their gradient-curvature motion. However, the amplitude of solar-wind driven ULF waves rapidly decreases with decreasing $L$. It is therefore not clear what mechanisms are responsible for fast transport rates observed inside the geosynchronous orbit. In this paper we investigate wether stormtime Pc5 waves can contribute to this process. Stormtime Pc5s have short azimuthal wave lengths and therefore cannot exhibit resonance with the the electron drift motion. However we show that stormtime Pc5s can cause localized random scattering of electron drift motion that violates the third invariant. According to our results electron interaction with stormtime Pc5s can produce rapid radial transport even as low as $L \simeq 4$. Numerical simulations show that electron transport can exhibit large deviations from radial diffusion. The diffusion approximation is not valid for individual storms but only applies to the statistically averaged response of the outer belt to stormtime Pc5 waves.
\end{abstract}

Keywords. Magnetospheric physics (Energetic particles, trapped) - Space plasma physics (Nonlinear phenomena; Wave-particle interactions)

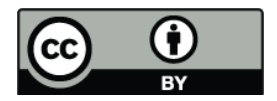

Correspondence to: A. Y. Ukhorskiy (ukhorskiy@jhuapl.edu)

\section{Introduction}

During intervals of increased geomagnetic activity relativistic electron fluxes in the outer radiation belt exhibit dynamic variability over multiple orders of magnitude. At the main phase of geomagnetic storms flux levels decrease drastically over a wide range of L-shells due to adiabatic effects and permanent losses to the magnetopause and the ionosphere (e.g., Millan and Thorne, 2007). In storm recovery, however, flux levels can increase to levels greatly exceeding their pre-storm values (e.g., Reeves et al., 2003). Since flux depletions at storm main phase are most likely dominated by permanent losses (Ukhorskiy et al., 2006a), the after-storm flux increases require radial transport and subsequent heating of energetic electrons from the outer to the inner magnetospheric regions.

Radial transport implies violation of the third adiabatic invariant associated with the drift motion of trapped electrons. In the absence of large disturbances, such as large-amplitude $(\sim 100 \mathrm{mV} / \mathrm{m})$ magnetosonic waves induced by interplanetary shock arrivals (Wygant et al., 1992), violation of the third invariant can be produced by quasi-periodic field disturbances over the course of multiple interactions with the electron drift motion. One widely accepted scenario is that the radial transport is driven by ULF oscillations of magnetic and/or electric fields in the Pc5 frequency band (period of $150-600 \mathrm{~s}, \omega \simeq 10-40 \mathrm{mHz}$ ) (Jocobs et al., 1964) that can break the third invariant in the process of drift resonance with electron motion:

$\omega-m\left\langle\omega_{D}\right\rangle=0$,

where $\omega$ is the frequency of oscillations, $\left\langle\omega_{D}\right\rangle$ is the bounce-averaged frequency of electron gradient-curvature drift around the Earth, and $m$ is the azimuthal wave number. The drift frequency of the outer belt electrons (kinetic energy between several hundred $\mathrm{keV}$ to few $\mathrm{MeV}$ ) varies in the range $\left\langle\omega_{D}\right\rangle \simeq 5-20 \mathrm{mHz}$. Thus, only the Pc5 pulsations

Published by Copernicus Publications on behalf of the European Geosciences Union. 


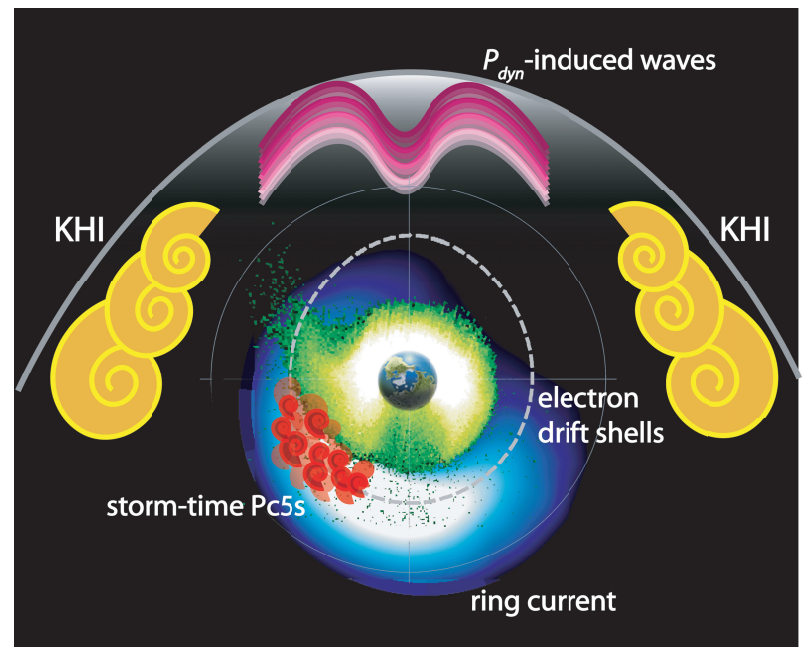

Fig. 1. Pc5 pulsations in the inner magnetosphere can be classified by their energy sources. The external pulsations are driven by solar wind variations at the day side magnetopause, while the internal pulsations are the result of low frequency instabilities of ring current ions.

with small $m$ numbers $(\lesssim 10)$ can exhibit drift resonance with the bulk of the outer belt electrons.

ULF pulsations in the inner magnetosphere can be broadly classified as externally and internally driven (see Fig. 1). The external ULF pulsations are attributed to variability of the solar wind parameters. Two main generation mechanisms suggested to account for the external pulsations are the KelvinHelmholz instability at the magnetopause flanks (e.g., Southwood, 1968) and compressions of the dayside magnetopause due to quasi-periodic variations in the solar wind dynamic pressure (e.g., Sarafopoulos, 1995; Kepko et al., 2002; Takahashi and Ukhorskiy, 2007). The solar-wind driven pulsations are most intense on the dayside magnetosphere (e.g., Ukhorskiy et al., 2005; Takahashi and Ukhorskiy, 2007) and generally have small $m$ numbers $(\lesssim 10)$ (e.g., Olson and Rostoker, 1978), which enables their drift resonant interaction with the outer belt electrons.

Previous theoretical analysis (e.g., Elkington et al., 2003; Ukhorskiy et al., 2005, 2006b; Ukhorskiy and Sitnov, 2008) showed that the solar-wind induced pulsations can provide an effective driver of radial transport in the vicinity of geosynchronous orbit. It is unlikely, however, that this mechanism remains efficient inside the geosynchronous orbit. The efficiency of a transpor mechanims in a given region of the belt (relative to other mechanisms acting in the region) can be assessed by comparing the characteristic timescales of the electron flux variability due this mechanim to the timescales determined from the locall spcecraft observations. On average radial transport due to the solar-wind driven pulsations can be approximated by a radial diffusion (Ukhorskiy and Sitnov, 2008). With the use of the locally linearized radial diffusion equation the characteristic time scales of order of magnitude variations of the flux can be estimated as: $\tau \simeq \tau_{D}(\delta L)^{2}$, where $\tau_{D}=1 / D_{L L}$ is the timescale given by the inverse diffusion coefficient and $\delta L$ is the characteristic spacial scale at which fluxes vary by a factor of 10 . From calcuations of the steadystate radial profiles of the flux (Shprits and Thorne, 2004), it was shown that at $L=4-5$ fluxes vary by an order of magnitude over $\delta L \lesssim 1$. Thus, in this region of the belt $\tau \lesssim \tau_{D}$. The radial diffusion coefficient of the sollar-wind driven pulsations strongly depends on the root-mean-square amplitude $E_{\varphi}^{\mathrm{rms}}$ of the azimuthal component of their electric field and the radial position $L$, as a result (e.g., Ukhorskiy et al., 2005):

$$
\tau_{D} \sim \frac{1}{L^{6}\left(E_{\varphi}^{\mathrm{rms}}\right)^{2}}
$$

Since energy sources of the external pulsations are at the magnetopause, their amplitude rapidly decreases with decreasing in $L$ (e.g., Mathie and Mann, 2001). Thus, a typical decrease of the root-mean-square amplitude from 0.5 to $0.1 \mathrm{mV} / \mathrm{m}$ (e.g., Ukhorskiy and Sitnov, 2008) leads to an increase of the characteristic radial transport time $\tau_{D}$ from 1 day at $L=7$ to 355 days at $L=4.5$. At the same time, it was observationally shown (Varotsou et al., 2008) that in the storm recovery phase electron fluxes at $L=4.2$ gain $1-2$ orders of magnitude on a timescale of 1-2 days. This suggests the existing action of other transport mechanisms providing fast flux enhancements inside the geosynchronous orbit.

The internal pulsations draw their energy from lowfrequency instabilities of ring current plasma (e.g., Hasegawa, 1969; Southwood, 1976). Being closely linked with injections of ring current ions, these pulsations have the occurrence rate maximum at storm main phase and thus are often referred to as "stormtime Pc5s" (Barfield and McPherron, 1978). Stormtime Pc5s have large azimuthal wave numbers $m=40-120$ (e.g., Takahashi et al., 1985), and therefore cannot exhibit drift resonance with the bulk of the energy distribution of the radiation belt electrons. Thus, in spite of the fact that on average in the inner magnetosphere stormtime Pc5s have amplitudes larger than the solar-wind driven pulsations, they are usually not included in the mechanisms considered in analysis of electron transport.

Enhancements of radiation belt fluxes do not directly correlate with the level of geomagnetic activity. Fluxes across wide $L$-ranges can increase even after minor geomagnetic storms $\left(D_{s t} \simeq-50 \mathrm{nT}\right)$. While this suggests that the radial transport is ubiquitous throughout the belt, it is not clear what mechanisms drive it inside geosynchronous orbit in the absence of relatively rare events such as associated with interplanetary shock arrivals (Hudson et al., 2000; Kress et al., 2007, 2008) or giant Pc5 waves (Lee et al., 2007).

A number of early studies of radial diffusion discussed various mechanisms that can transport electrons across the belt in the absence of the drift resonance. The proposed non-resonant mechanisms such as sporadic magnetopause 
compressions due to variations in the solar wind dynamic pressure (Parker, 1960) or random fluctuations in the convection electric field (Schultz and Lanzerotti, 1974) are mostly active at the outer edge of the belt and therefore can not be efficient well inside the geosynchronous region. In this paper we investigate whether stormtime Pc5 pulsations should be considered as a viable non-resonant mechanism of radial transport in the outer radiation belt. The study was motivated by the following. First, early observational analysis (e.g., Lanzerotti et al., 1969) established correlation of stormtime Pc5 power and electron heating. Second, large-amplitude $(\gtrsim 10 \mathrm{mV} / \mathrm{m})$ high- $m$ Pc5 pulsations are observed well inside the geosynchronous orbit $(L \sim 4.5)$ (e.g., Eriksson et al., 2006; Ohtani et al., 2007). Finally, drift resonance is not necessary for violation of the third adiabatic invariant. Indeed, the invariant of a quasi-periodic particle motion can be violated, if the perturbation fields vary on spatial scales comparable or smaller than the orbit size. Scattering at such localized fields can create sharp kinks in particle trajectories and break the invariant. For example, it is well known that the first adiabatic invariant of a particle is violated when its Larmor radius is comparable to the radius of the magnetic field curvature (e.g., Birmingham, 1984). Stormtime Pc5s are a potential candidate for local violation of the third invariant of trapped electrons since their wavelength is substantially smaller than the electron drift orbit scales.

With the use of analytical estimates and test-particle simulations we show that stormtime Pc5s can be an efficient driver of radial transport in the bulk of the outer belt. It is the combination of the spatial and temporal properties of the wave fields that enables the transport. Random distribution of wave electric fields across the wave activity region produces radial scattering of electrons drifting through the wave. Due to temporal evolution of the wave fields different particles in the region get scattered by different random fields. This produces phase mixing in their drift motion and as a result their collective motion across the drift shells becomes stochastic. It is also suggested that due to the complex interplay of characteristic spatial and temporal scales in the system, the transport is likely to exhibit large deviations from radial diffusion, the approximation commonly used in radiation belt models (e.g., Shprits et al., 2008).

The paper is organized as following. In the next section we describe stormtime Pc5 activity observed during high variability of radiation belt fluxes. In Sect. 3 we present our test-particle model and analytical estimates of electron interaction with stormtime Pc5s. The results of our numerical simulations are discussed in Sect. 4 followed by the conclusions.

\section{Distribution of stormtime Pc5s}

To survey variability of the Pc5 wave power during radiation belt storms we used field and particle data from the
Combined Release and Radiation Effects Satellite (CRRES) (Johnson and Kierein, 1992). We selected a 20-day interval from the late phase of the mission. In that timeframe the spacecraft sampled the afternoon sector, where the ULF wave activity is believed to be dominated by stormtime Pc5s (e.g., Takahashi and Anderson, 1992). Figure 2 shows an overview of the wave data along with geomagnetic conditions and electron fluxes. The $L$ distribution of the perpendicular fluxes of $>0.5 \mathrm{MeV}$ electrons measured by the MEA detector is shown in the bottom-left panel of the figure. The selected period contained five major geomagnetic storms $(\mathrm{SymH}<-100 \mathrm{nT})$. The storm-time electron fluxes varied by orders of magnitude over a broad $L$ range including the outer belt, the inner belt and the slot region. The root-mean-square amplitude of electric field variations with period less than $10 \mathrm{~min}$ is shown in the middle-left panel. During selected storms the amplitude exceeded $1 \mathrm{mV} / \mathrm{m}$ even at $L$ as low as 3. Thus, variability of radiation belt fluxes is accompanied by increased stormtime Pc5 activity extended over the bulk of the outer belt, well inside the geosynchronous region.

It has to be noted, that to compute the $L-U T$ distribution of the wave amplitude shown in Fig. 2, the root-mean-square amplitude of the electric field in the ULF fluctuations was averaged over time periods as long as $15 \mathrm{~h}$. The peak-to-peak amplitude during individual wave events can be substantially larger than $1 \mathrm{mV} / \mathrm{m}$. The right panel of the figure shows a two-hour interval of electric and magnetic field variations from the inbound pass of CRRES on DOY 163. Substantial Pc5 activity was observed from $L$ of 6 to 4 with maximum peak-to-peak amplitude of the azimuthal electric field variations of $5 \mathrm{mV} / \mathrm{m}$ at $L \simeq 5.5$.

\section{Electron interaction with stormtime Pc5s}

The efficiency of electron scattering by stormtime Pc5s is determined by the field-aligned structure of the waves. The bounce frequency of relativistic electrons $(1-10 \mathrm{~Hz})$ greatly exceeds the frequency of Pc5 waves. Consequently, fieldaligned profiles of the wave fields can be considered stationary on the timescales of the electron bounce motion. Thus, if the wave electric field is asymmetric in respect to the magnetic equator, the net change in the electron energy over a pass through the wave activity on the course of its bounce motion is zero. On the other hand, waves with symmetric latitudinal profiles of transverse electric fields can produce a net change of electron energy.

Stormtime Pc5 waves with both symmetric and asymmetric field-aligned structures are observed in the magnetosphere. Waves with asymmetric structures (Takahashi et al., 1987) are believed to be generated by the drift-mirror instability of ring current ions (Chen and Hasegawa, 1974; Southwood, 1976), while symmetric structures (Takahashi et al., 1987; Takahashi and Anderson, 1992; Eriksson et al., 2006) were explained by the nonlinear frequency doubling effects 

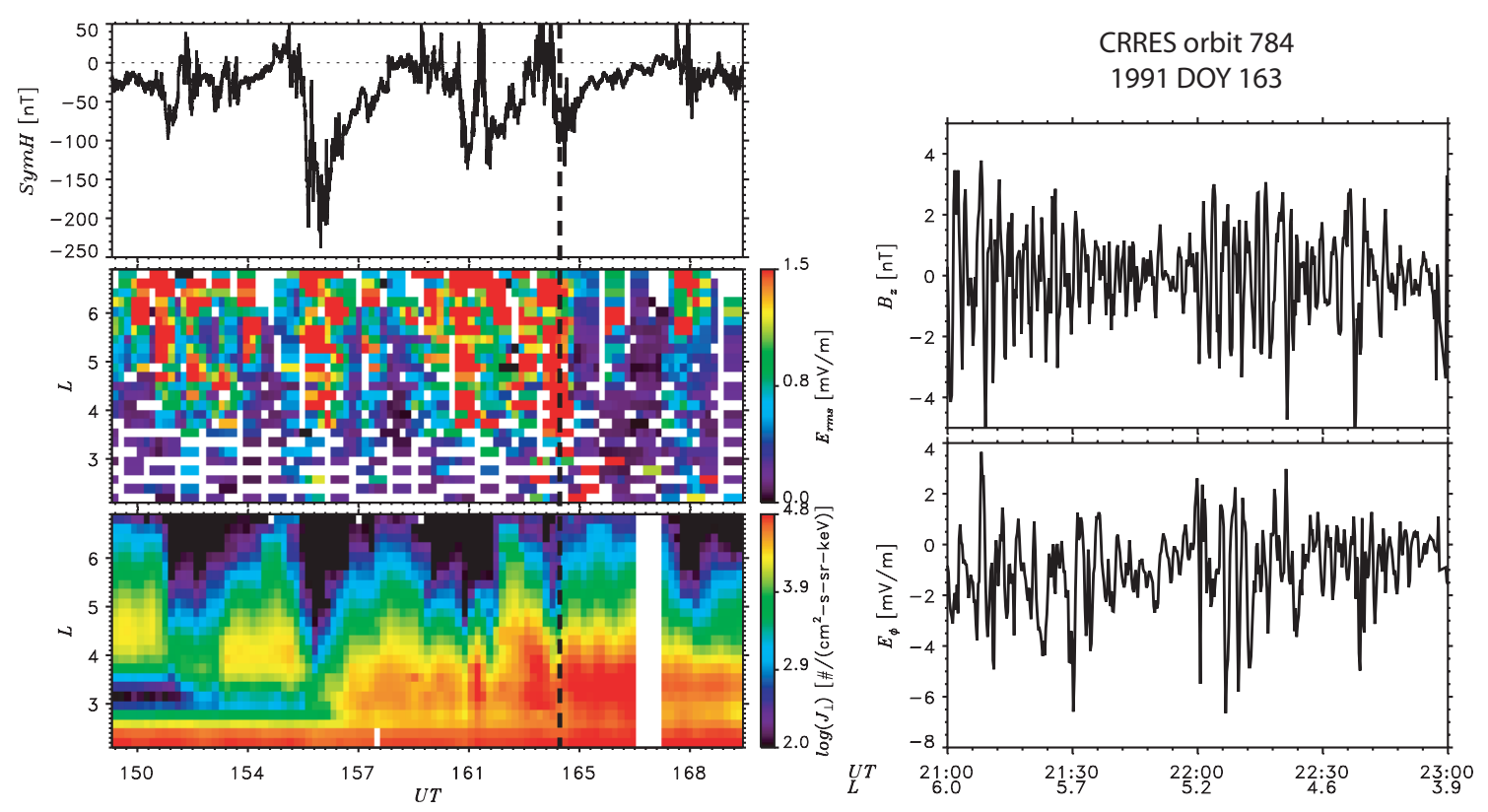

Fig. 2. Left panel: A survey of stormtime Pc5 (period $<10 \mathrm{~min}$ ) wave activity and variations of relativistic $(>0.5 \mathrm{MeV})$ electron fluxes measured by CRRES, plotted as a function of time and $L$. Right panel: $E_{\varphi}$ and $B_{z}$ oscillations associated with a stormtime Pc5 pulsation on DOY 163 (dashed line in the left panel).

(Southwood and Kivelson, 1997) and the drift-compressional instability (Cheng and Lin, 1987). Recently Ohtani et al. (2007) reported observations of high- $m$ Pc5 waves with electric field amplitude as high as a few tens of $\mathrm{mV} / \mathrm{m}$ as low as at $r=4.6 R_{E}$. Since these waves were observed close to the magnetic equator $\left(\left|Z_{S M}\right|<1 R_{E}\right)$, it is plausible that they have symmetric field-aligned structure and therefore can radially scatter relativistic electrons in the center of the outer belt.

To estimate the efficiency of electron scattering by stormtime Pc5 waves with symmetric field-aligned electric field structure, a test particle approach was used. The wavelengths of stormtime Pc5s greatly exceed the Larmor radius and their period is much greater than the gyro-period of relativistic electrons. The waves, therefore, do not break the first invariant of radiation belt electrons and the electron motion can be considered in the guiding center approximation. The electric fields of stormtime Pc5s do not have parallel component and their magnetic field is stationary on the timescales the electron bounce motion. Thus, electron interaction with stormtime Pc5s conserves their second invariant. Since the waves also are mostly confined to the equatorial regions $\left(<20^{\circ}\right)$ (Takahashi et al., 1987), the consideration was restricted to electrons bouncing at the magnetic equator. The guiding center motion of equatorial electrons in the presence of stormtime Pc5s is dominated by the gradient-curvature drift in the leading dipole component of the geomagnetic field and radial motion due to the azimuthal electric field $E_{\varphi}$ of the waves (e.g., Ukhorskiy et al., 2005):
$\left\{\begin{array}{l}R_{E} \dot{L}=c \frac{E_{\varphi}}{B_{0}} L^{3} \\ \dot{\varphi}=\omega(L)\end{array}\right.$,

where $\varphi$ is the azimuthal angle, $R_{E}$ is the Earth's radius, $B_{0}$ is the magnetic field magnitude at the Earth's surface at the equator, $c$ is the speed of light, and $\omega=\frac{3 \mu c}{\gamma e} \frac{1}{\left(R_{E} L\right)^{2}}$ is the frequency of the gradient-curvature drift.

Stormtime Pc5s are considered to be uncorrelated ripples of slow magnetosonic mode (e.g., Sonnerup et al., 1969) drifting westward along with their energy source, the ring current (10-100 keV) ions (Takahashi et al., 1985). The following properties of the waves were assumed for modeling their impact on electron motion:

1. The wave activity covers a limited region $(\Delta \varphi)$ on the duskside inner magnetosphere corresponding to the extent of the instability responsible for the wave growth.

2. The wave activity consists of random field structures with the azimuthal extent $\delta \varphi=2 \pi / m$ and varying radial extent $\delta L$, such that $\delta L^{\mathrm{rms}}=L \delta \varphi$ (see left panel of Fig. 5).

3. The field amplitudes at different structures of the wave activity are distributed randomly, such that their azimuthal electric field, $E_{k}$, satisfies: $\left\langle E_{k}\right\rangle=0$, $\left\langle\left(E_{k}\right)^{2}\right\rangle=\left(E^{\mathrm{rms}}\right)^{2}$, where $E^{\mathrm{rms}}$ is the observed value of the root-mean-square amplitude of stormtime Pc5s. 

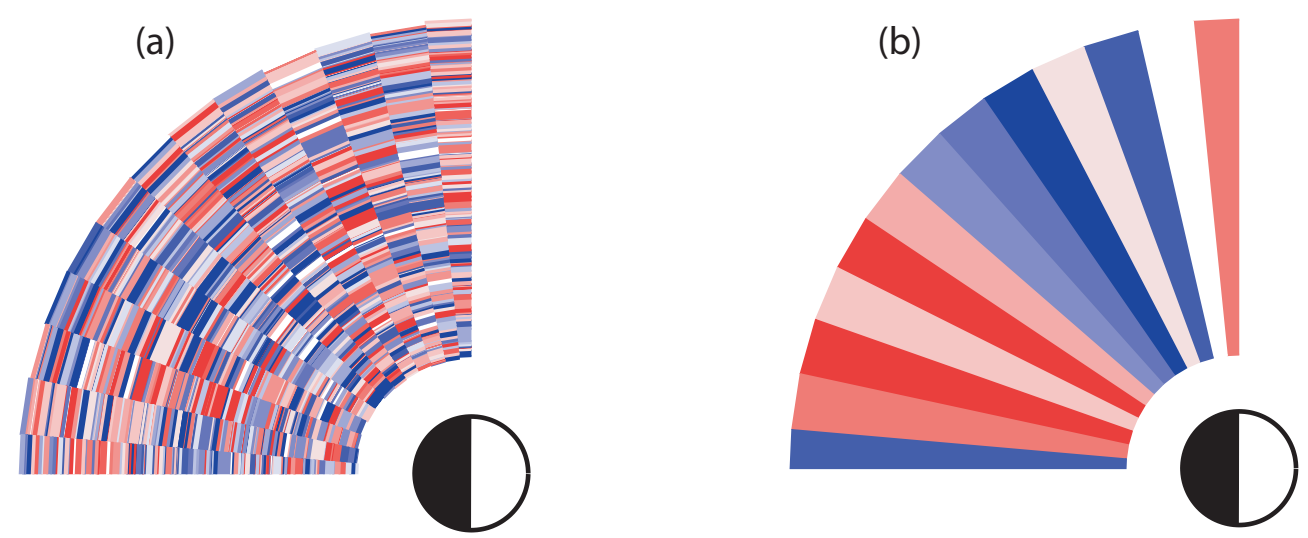

Fig. 3. A schematic illustration of two extreme limits of radial distribution of the electric field structures in stormtime Pc5s: (a) $\delta L^{\mathrm{rms}} \rightarrow 0$ and (b) $\delta L^{\mathrm{rms}} \rightarrow L_{0}$ limits.

4. The structures drift in the westward direction at the speed of the gradient-curvature drift of ring current ions $\left(\omega_{i}\right)$. The wave activity is maintained due to new ring current ions injected into the region. Thus, while the structures disappear as they reach the westward end of the region, new structures appear at the eastward end.

Radial transport in the system is quantified by the first two moments of the electron distribution function. The moments can be estimated from the guiding center trajectories $\Delta L_{i}(t)$ of an ensemble of $N$ test particles starting their motion at a common radial position $L_{0}$ (e.g., Ukhorskiy and Sitnov, 2008):

$$
\begin{aligned}
& \langle\langle\Delta L(t)\rangle\rangle=\frac{1}{N} \sum_{i=1}^{N}\left(L_{i}(t)-L_{0}\right) \\
& \left\langle\left\langle(\Delta L(t))^{2}\right\rangle\right\rangle=\frac{1}{N} \sum_{i=1}^{N}\left(\Delta L_{i}(t)-\langle\langle\Delta L(t)\rangle\rangle\right)^{2} .
\end{aligned}
$$

If at a given $L_{0}\langle\langle\Delta L(t)\rangle\rangle=0$ and $\left\langle\left\langle(\Delta L(t))^{2}\right\rangle\right\rangle=2 D_{L L} t$, transport is a radial diffusion process.

To elucidate the properties of electron transport in stormtime Pc5 fields we consider two extreme limits of the wave parameter values: (1) $\delta L^{\mathrm{rms}} \rightarrow 0$ and (2) $\delta L^{\mathrm{rms}} \rightarrow L_{0}$ (see Fig. 3). According to the first equation of system (3), the radial position of an electron after its pass through a single wave structure changes by $\frac{c}{B_{0} R_{E}} L_{0}^{3} \tau E_{k}$, where $\tau=\delta \varphi / \omega_{0}$ is the time it takes the electron to drift through the structure. Since in the first case $\delta L^{\mathrm{rms}} \rightarrow 0$, an electron interacts with a given structure of the wave activity only once. After its pass through the structure it moves radially into the spatial domain of a new structure, where its radial position changes again. Since the electric field values $E_{k}$ at different structures are distributed randomly, an electron, uniformly rotating in azimuth, exhibits random walk in the radial direction. This motion can be described by the following algebraic map:
$\left\{\begin{array}{l}L(t+T)=L(t)+\frac{c}{B_{0} R_{E}} L_{0}^{3} \tau E_{k} \\ \varphi(t+T)=\varphi(t)+\omega_{0} T\end{array}\right.$,

where the time step $T$ is the time between the consecutive interactions with the wave structures: $T=T_{\min }=\tau$.

In the second case $\delta L^{\mathrm{rms}} \rightarrow L_{0}$ and electrons at a given azimuth interact with the same wave structure regardless of their radial position. Since electrons drift much faster than the wave structures $\left(\omega_{0} \gg \omega_{i}\right)$, an electron passing through a given azimuthal location of the wave activity, interacts with the same structure over multiple drift periods, until the structure shifts westward and a new structure moves in its place. This results in a random scattering of the electron in radial direction. Thus, similarly to the previous case the electron motion is described by map (5). The time step of the map, however, is now determined by the azimuthal drift speed of wave structures: $T=T_{\max }=\delta \varphi / \omega_{i}$, rather than the electron drift motion.

In both of the described cases different particles of an ensemble of electrons initially located at some $L_{0}$ interact with different random sectors of the wave activity at every time step of the map. Each particle of the ensemble exhibits a random walk in radial direction. Since the sequence of random steps of this walk is different for different particles, phase correlations between particles decay in time. On time scales longer than the correlation decay time the collective electron motion becomes a radial diffusion process with $\langle\langle\Delta L(t)\rangle\rangle=0$. The diffusion coefficient in this case can be estimated from the phase average a single step of the map (e.g., Lichtenberg and Lieberman, 1983) as:

$D_{L L}=\frac{\left\langle\left\langle(\Delta L(T))^{2}\right\rangle\right\rangle}{2 T}$,

where $\langle\langle\cdots\rangle\rangle=\frac{1}{2 \pi} \int_{0}^{2 \pi} \cdots d \varphi$. 


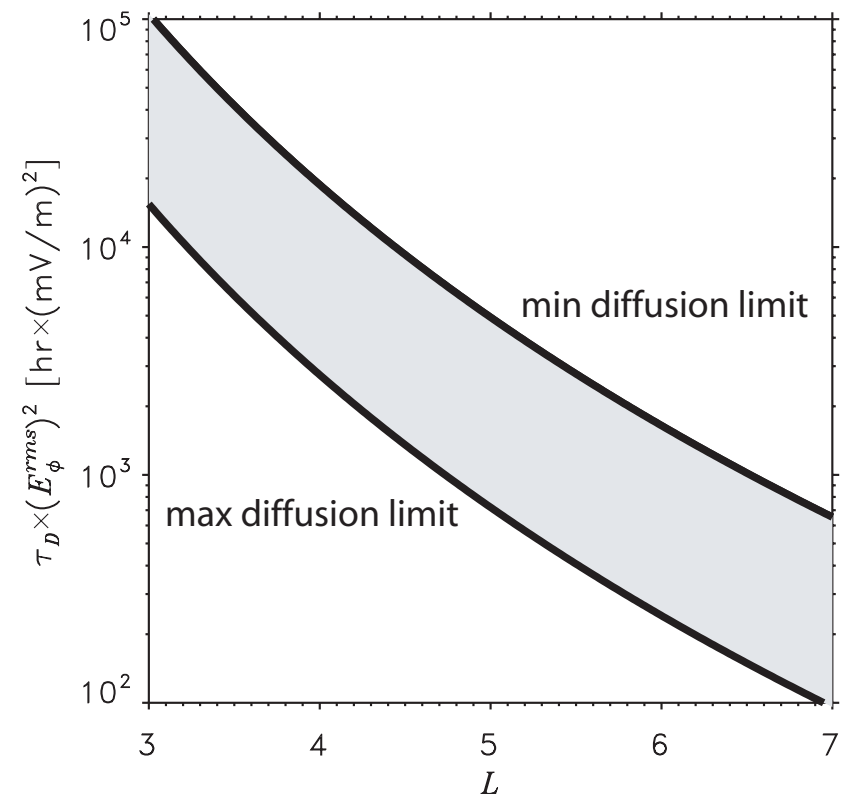

Fig. 4. Characteristic timescales of radial transport due to stormtime Pc5s normalized to the root-mean-square amplitude of the wave electric field.

In the case of realistic wave parameters, e.g. when the radial extent $\delta L$ of the wave structures is finite, the electron transport is more complicated. Electrons can exhibit random scattering in radial direction due to either their radial displacement from one wave structure to another or due to the phase shift of the wave structures relative to the azimuthal electron motion. The time interval between consecutive interactions in this case is no longer constant and the electron motion can no longer be described by algebraic map (5) with a constant time step.

Another complication results from large separation of the characteristic timescales in the system: electron scattering in radius is much slower than the azimuthal drift of the wave structures which is much slower than the electron drift motion. Indeed, consider two groups of particles drifting through a wave activity region. If both groups start at the same initial position $L_{0}$, over one drift period the particles of both groups interact with the same set of wave structures such that $\langle\langle\Delta L\rangle\rangle=0$. If, however, the groups are radially separated by $\sim \delta L$, particles of different groups interact with different sets of structures and as a result the average over both groups of particles $\langle\langle\Delta L\rangle\rangle$ is no longer zero. Thus it can be expected that while electrons of the ensemble separate in radius, $\langle\langle\Delta L\rangle\rangle$ can exhibit large deviations from zero. It may result in long-term correlations in radial motion of different groups of particles and cause substantial deviations from radial diffusion.

While the above extreme limits leading to map (5) do not describe the details of radial transport with realistic wave pa- rameters, they can be used as estimates of the lower and the upper limits of radial transport in the system. From Eqs. (5) and (6) it follows that the diffusion coefficients in these cases are equal to:

$$
\begin{aligned}
\left\{\begin{array}{c}
D_{L L}^{\max } \\
D_{L L}^{\min }
\end{array}\right\} & =\frac{\left\langle\left\langle(\Delta L)^{2}\right\rangle\right\rangle}{2}\left\{\begin{array}{c}
\frac{1}{T_{\min }} \\
\frac{1}{T_{\max }}
\end{array}\right\} \\
& =\frac{\Delta \varphi}{2 m}\left(\frac{c}{R_{E} B_{0}}\right)^{2} \frac{L_{0}^{6}}{\omega_{0}} E_{\mathrm{rms}}^{2}\left\{\begin{array}{c}
1 \\
\frac{\omega_{i}}{\omega_{0}}
\end{array}\right\} .
\end{aligned}
$$

Figure 4 shows the timescales of radial transport $\tau_{D}=1 / D_{L L}$ as function of $L$ calculated from Eq. (7) for $m=50$ and $\Delta \varphi=\pi / 2$. The timescales were normalized by the rootmean-square amplitude of the wave electric field. According to these estimates large-amplitude Pc5 waves can be an efficient driver of transport even al low $L$ shells. Thus, a $10 \mathrm{mV} / \mathrm{m}$ wave can drive radial transport with timescales as short as $30 \mathrm{~h}$ at $L$-values as low as 4 . Ohtani et al. (2007) reported CLUSTER observations of a $10 \mathrm{mV} / \mathrm{m}$ ULF wave activity over a time period of $\sim 1 \mathrm{~h}$, when the spacecraft was near the equator. Whether such high-amplitude wave activity can persist on timescales $\sim 1$ day, needs to be determined by the future observational analysis of stormtime Pc5 waves.

\section{Numerical simulations of radial transport}

To verify our analytical estimates and to investigate possible deviations of transport from radial diffusion we conducted a series of numerical test-particle simulations. The simulations were based on Eq. (3) and therefore were not restricted by simplified assumptions used in derivation of map (4). Electron motion was computed in wave fields with moderate amplitude $E_{\varphi}^{\text {rms }}=2 \mathrm{mV} / \mathrm{m}$ and azimuthal wave number $m=50$ extended over $\Delta \varphi=\pi / 2$ and other properties discussed in Sect. 3. Radial transport in the simulations was quantified by the second moment of radial distribution function (5) calculated with the use of an ensemble of $10^{3}$ electrons with the initial position $L_{0}=5$.

The results are summarized in Fig. 5. Panel (a) shows a snapshot of electron motion in the presence of stormtime Pc5s. Particles are shown with circles and the wave electric field is indicated by color. Panel (b) shows time dependence of the second moment of radial distribution functions computed from the simulation results. Black curves show $\left\langle\left\langle\left(\Delta L_{k}(t)\right)^{2}\right\rangle\right\rangle$ for 30 different realizations of electron motion in statistically identical wave fields. The only difference among realizations was in random distributions of electric field values between individual structures of the wave activity. As can be seen from the figure, $\left\langle\left\langle\left(\Delta L_{k}(t)\right)^{2}\right\rangle\right\rangle$ from different realizations differ substantially from each other. They also exhibit non-monotonic dependence on time inconsistent with diffusive transport, in which the second moment $\left\langle\left\langle(\Delta L(t))^{2}\right\rangle\right\rangle$ scales as $t^{\beta}$, where $\beta$ may deviate from 1 in the 

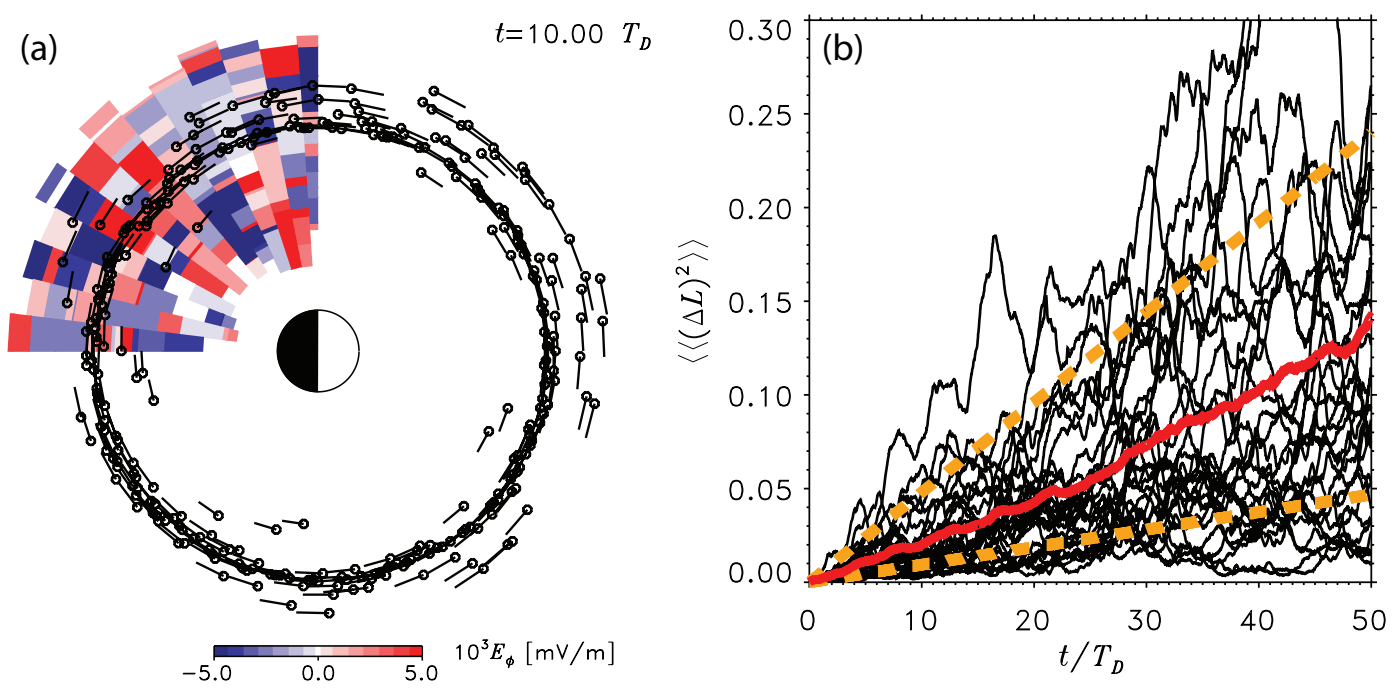

Fig. 5. Panel (a) A snapshot of electron motion in the presence of stormtime Pc5s: $E_{\varphi}^{\mathrm{rms}}=2 \mathrm{mV} / \mathrm{m}, m=50$, and $\Delta \varphi=\pi / 2$. Panel (b) Radial transport in 30 statistically similar realizations of electron motion (black lines), analytical estimates of the upper and lower limits of radial diffusion (yellow line). Radial transport averaged over all realizations (red line).

case of anomalous diffusion and when diffusion coefficient is not a constant (e.g. Zaslavsky, 2002).

The lower and the upper diffusion limits given by estimates (7) are shown in yellow. Most of the $\left\langle\left\langle\left(\Delta L_{k}(t)\right)^{2}\right\rangle\right\rangle$ curves are confined between the lower and the upper diffusion limits predicted by our analytical estimates. The red line shows radial transport averaged over all realizations: $\frac{1}{N_{r}} \sum_{k=1}^{N_{r}}\left\langle\left(\Delta L_{k}(t)\right)^{2}\right\rangle$. Its approximately linear time dependence means that while individual events can exhibit large deviations from radial diffusion, on average, transport due to stormtime Pc5s is a diffusion process. The fact that the average transport line falls in between the estimates of the lower and the upper diffusion limits suggests that our analytical estimates (7) can be used for evaluating the timescales of electron transport in stormtime Pc5 waves.

\section{Conclusions}

A test-particle approach was used to address the role of stormtime Pc5 waves in radial transport of relativistic electrons in the outer radiation belt. We were particularly interested in whether stormtime Pc5s can drive electron transport inside geosynchronous orbit where the amplitudes of solarwind driven ULF waves are too low to explain the observed transport rates.

It was shown that stormtime Pc5 waves with symmetric field-aligned structure can effectively interact with radiation belt electrons and cause their stochastic scattering across the drift shells. Since stormtime Pc5s have high azimuthal wave numbers $(m=40-120)$ they cannot exhibit resonance with the electron drift motion. The onset of stochasticity in this case has a different origin than in the case of electron interaction with low- $m$ ULF waves, which can be in the drift resonance with relativistic electrons. When particles resonantly interact with waves, their motion can become stochastic even if the wave fields are regular. Stochasticity then results form the overlap of electron populations in resonance with adjacent harmonics of the wave field. In the absence of resonance waves still can produce stochastic scattering of particles, if the wave fields themselves are random. In the case of stormtime Pc5s, stochastic scattering and subsequent radial transport is a result of random distribution of the electric field across the wave activity region and its complex temporal evolution.

Based on the observed properties of stormtime Pc5s we developed a model in which the wave field was represented as a set of uncorrelated structures of electric field oscillations drifting with their energy source, energetic $(10-100 \mathrm{keV})$ ring current ions. We showed that the lower and the upper limits of radial transport in this model can be described as radial diffusion and derived analytical expressions for the corresponding diffusion coefficients. The derived radial diffusion coefficients can be used for analysis of the characteristic timescales of electron flux variations due to stormtime Pc5s. In particular, it was shown that large-amplitude waves $(\sim 10 \mathrm{mV} / \mathrm{m})$, previously observed in space, can produce order of magnitude variations in the flux as low as $L=4$ on timescales as short as $30 \mathrm{~h}$, which is in agreement with observational estimates of the flux rise times. This identifies stormtime Pc5 waves as a potentially important mechanism of the radiation belt flux variability inside the geosynchronous orbit. 
Numerical simulations of electron motion in the presence of stormtime Pc5 waves showed that for realistic values of wave parameters electron transport can exhibit large deviations from radial diffusion. It was also shown that the deviations disappear when the transport is averaged over multiple realizations. This suggests that radial diffusion approximations can be used in the analysis of statistically average response of the electron belt to stormtime Pc5 activity.

It has to be noted, that as in any test-particle simulations the results of our numerical experiments were determined by properties of the fields driving electron motion. Since the existing physics-based models of the inner magnetosphere cannot describe stormtime Pc5 waves, we used an empirical model based an ad hoc representation motivated by the observed characteristics of the waves. However, not all the properties of stormtime Pc5s, affecting their interaction with relativistic electrons, are well known. Further observational studies are necessary to refine our understanding of stormtime Pc5 waves and critically assess whether the natural system actually behaves in a manner that our initial studies suggest.

Acknowledgements. The research is supported by the NSF grant ATM-0540121.

Topical Editor I. A. Daglis thanks two anonymous referees for their help in evaluating this paper.

\section{References}

Barfield, J. N. and McPherron, R. L.: Stormtime Pc 5 magnetic pulsations observed at synchronous orbit and their correlation with the partial ring current, J. Geophys. Res., 83, 739-743, 1978.

Birmingham, T. J.: Pitch angle diffusion in the Jovian magnetodisc, J. Geophys. Res., 89, 2699-2707, 1984.

Chen, L. and Hasegawa, A.: A theory of long-period magnetic pulsation, J. Geophys. Res., 79, 1024-1032, 1974.

Cheng, C. Z. and Lin, C. S.: Eigenmode analysis of compressional waves in the magnetosphere, Geophys. Res. Lett., 14, 884-887, 1987.

Elkington, S. R., Hudson, M. K., and Chan, A. A.: Resonant acceleration and diffusion of outer zone electrons in an asymmetric geomagnetic field, J. Geophys. Res., 108, 1116, doi:10.1029/2001JA009202, 2003.

Eriksson, P. T. I., Blomberg, L. G., and Glassmeier, K. H.: Cluster satellite observations of $\mathrm{mHz}$ pulsations in the dayside magnetosphere, Adv. Space Res., 38, 1730, doi:10.1016/j.asr.2005.04.103, 2006.

Hasegawa, A.: Drift mirror instability in the magnetosphere, Phys. Fluids, 12, 2642-2650, 1969.

Hudson, M. K., Elkington, S. R., Lyon, J. G., and Goodrich, C. C.: Increase in relativistic electron flux in the inner magnetosphere: ULF wave mode structure, Adv. Space Res., 25, 2327-2337, 2000.

Jocobs, J. A., Kato, Y., Matsushita, S., and Troitskaya, V. A.: Classification of geomagnetic micropulsations, J. Geophys. Res., 69, 180-181, 1964.
Johnson, M. H. and Kierein, J.: Combined Release and Radiation Effects Satellite (CRRES): Spacecraft and mission, J. Spacecr. Rockets, 29, 556-563, 1992.

Kepko, L., Spence, H. E., and Singer, H. J.: ULF waves in the solar wind as direct drivers of magnetospheric pulsations, Geophys. Res. Lett., 29(8), 1197, doi:10.1029/2001GL014405, 2002.

Kress, B., Hudson, M., Looper, M., Lyon, J., and Goodrich, C.: Global MHD test particle simulations of solar energetic electron trapping in the Earth's radiation belts, J. Atmos. Solar Terr. Phys., 70, 1727, doi:10.1016/j.jastp.2008.05.018, 2008.

Kress, B. T., Hudson, M. K., Looper, M. D., Albert, J., Lyon, J. G., and Goodrich, C. C.: Global MHD test particle simulations of $>10 \mathrm{MeV}$ radiation belt electrons during storm sudden commencement, J. Geophys. Res., 112, A09215, doi:10.1029/2006JA012218, 2007.

Lanzerotti, L. J., Hasegawa, A., and Maclennan, C. G.: Drift mirror instability in the magnetosphere: Particle and field oscillations and electron heating, J. Geophys. Res., 74, 5565-5578, 1969.

Lee, E. A., Mann, I. R., Lotoaniu, T., and Dent, Z. C.: Global Pc5 pulsations observed at unusually low $\mathrm{L}$ during the great magnetic storm of 24 March 1991, J. Geophys. Res., 112, A05208, doi:10.1029/2006JA011872, 2007.

Lichtenberg, A. J. and Lieberman, M. A.: Regular and Chaotic Dynamics, vol. 38 of Applied Mathematical Sciences, SpringerVerlag, New York, 2 edn., 1983.

Mathie, R. A. and Mann, I. R.: On the solar wind control of Pc5 ULF pulsation power at mid-latitudes: Implications for $\mathrm{MeV}$ electron acceleration in the outer radiation belt, J. Geophys. Res., 106, 29783-29796, 2001.

Millan, R. M. and Thorne, R. M.: Review of radiation belt relativistic electron losses, J. Atmos. Solar Terr. Phys., 69, 362, doi:10.1016/j.jastp.2006.06.019, 2007.

Ohtani, S., Korth, H., Brandt, P. C., Blomberg, L. G., Singer, H. J., Henderson, M. G., Lucek, E. A., Frey, H. U., Zong, Q., Weygand, J. M., Zheng, Y., and Lui, A. T. Y.: Cluster observations in the inner magnetosphere during the 18 April 2002 sawtooth event: Dipolarization and injection at $r=4.6 R_{E}$, J. Geophys. Res., 112, A08213, doi:10.1029/2007JA012357, 2007.

Olson, J. V. and Rostoker, G.: Longitudinal phase variations of Pc 4-5 micropulsations, J. Geophys. Res., 83, 2481-2488, 1978.

Parker, E. N.: Geomagnetic fluctuations and the form of the outer zone of the Van Allen radiation belt, J. Geophys. Res., 65, 31173130, 1960.

Reeves, G. D., McAdams, K. L., Friedel, R. H. W., and O'Brien, T. P.: Acceleration and loss of relativistic electrons during geomagnetic storms, Geophys. Res. Lett., 30(10), 1529, doi: 10.1029/2002GL016513, 2003.

Sarafopoulos, D. V.: Long duration Pc 5 compressional pulsations inside the Earth's magnetotail lobes, Ann. Geophys., 13, 926937, 1995, http://www.ann-geophys.net/13/926/1995/.

Schultz, M. and Lanzerotti, L. J.: Particle Diffusion in the Radiation Belts, vol. 7 of Physics and Chemistry in Space, Springer-Verlag, Berlin, Heidelberg, New York, 1974.

Shprits, Y. Y. and Thorne, R. M.: Time dependent radial diffusion modeling of relativistic electrons with realistic loss rates, Geophys. Res. Lett., 31, L08805, doi:10.1029/2004GL019591, 2004.

Shprits, Y. Y., Elkington, S. R., Meredith, N. P., and Subbotin, D. A.: Review of modeling of losses and sources of relativistic 
electrons in the outer radiation belt: 1. Radial transport, J. Atmos. Solar-Terr. Phys., 70, 1679, doi:10.1016/j.jastp.2008.06.008, 2008.

Sonnerup, B., Cahill, L. J., and Davis, L. R.: Resonant vibration of the magnetosphere observed from Explorer 26, J. Geophys. Res., 74, 2276-2287, 1969.

Southwood, D. J.: The hydromagnetic stability of the magnetopause boundary, Planet. Space Sci., 16, 587-605, 1968.

Southwood, D. J.: A general approach to to low-frequency instability in the ring current plasma, J. Geophys. Res., 81, 3340-3348, 1976.

Southwood, D. J. and Kivelson, M.: Frequency doubling in ultralow frequency wave signals, J. Geophys. Res., 102(A12), 2715127158, 1997.

Takahashi, K. and Anderson, B. J.: Distribution of ULF energy $(f<80 \mathrm{mHz})$ in the inner magnetosphere: A statistical analysis of AMPTE CCE magnetic field data, J. Geophys. Res., 97, 1075110773, 1992.

Takahashi, K. and Ukhorskiy, A. Y.: Solar wind control of Pc5 pulsation power at geosynchronous orbit, J. Geophys. Res., 112, A11205, doi:10.1029/2007JA012483, 2007.

Takahashi, K., Higbie, P. R., and Baker, D. N.: Azimuthal propagation and frequency charecteristic of compressional Pc 5 waves observed at geostationary orbit, J. Geophys. Res., 90, 14731485, 1985.

Takahashi, K., Fennell, J. F., Amata, E., and Higbie, P. R.: Field aligned structure of the storm time Pc 5 wave of November 1415, 1979, J. Geophys. Res., 92, 5857-5864, 1987.
Ukhorskiy, A. Y. and Sitnov, M. I.: Radial transport in the outer radiation belt due to global magnetospheric compressions, J. Atmos. Solar-Terr. Phys., 70, 1714, doi:10.1016/j.jastp.2008.07.018, 2008.

Ukhorskiy, A. Y., Takahashi, K., Anderson, B. J., and Korth, H.: The impact of toroidal ULF waves on the outer radiation belt electrons, J. Geophys. Res., 110, A10202, doi:10.1029/2005JA011017, 2005.

Ukhorskiy, A. Y., Anderson, B. J., Brandt, P. C., and Tsyganenko, N. A.: Storm-time evolution of the outer radiation belt: Transport and losses, J. Geophys. Res., 111, A11S03, doi:10.1029/2006JA011690, 2006a.

Ukhorskiy, A. Y., Takahashi, K., Anderson, B. J., and Tsyganenko, N. A.: The impact of ULF oscillations in solar wind dynamic pressure on the outer radiation belt electrons, Geophys. Res. Lett., 33, L06111, doi:10.1029/2005GL024380, 2006 b.

Varotsou, A., Friedel, R. H., Reeves, G. D., Lavraud, B., Skoug, R. M., Cayton, T. E., and Bourdarie, S.: Characterization of relativistic electron flux rise times during the recovery phase of geomagnetic storms as measured by the NS41 GPS satellite, J. Atmos. Solar-Terr. Phys., 70, 1745, doi:10.1016/j.jastp.2008.01.020, 2008.

Wygant, J. R., Harvey, P. R., Pankow, D., Mozer, F. S., Maynard, N., Singer, H., Smiddy, M., Sullivan, W., and Anderson, P.: CRRES electric field/Langmuir probe instrument, J. Spacecraft and Rockets, 29, 601-604, 1992.

Zaslavsky, G. M.: Chaos, fractional kinetics, and anomalous transport, Physics Reports, 371, 461, 2002. 